\title{
Correlation of Sperm Parameters With Semen Lipid Peroxidation and Total Antioxidants Levels in Astheno- and Oligoasheno-Teratospermic Men
}

\author{
Abasalt Hosseinzadeh Colagar ${ }^{1, *}$, Fatemeh Karimi ${ }^{1}$, Seyed Gholam Ali Jorsaraei ${ }^{2}$ \\ 1 Department of Molecular and Cell Biology; Nano and Biotechnology Research Group, Faculty of Basic Sciences, University of Mazandaran, Babolsar, IR Iran \\ 2 Fatemeh-Zahra Infertility and Health Reproductive Research Center, Babol University of Medical Sciences, Babol, IR Iran \\ ${ }^{*}$ Corresponding author: Abasalt Hosseinzadeh Colagar, Department of Molecular and Cell Biology, Nano and Biotechnology Research Group, Faculty of Basic Sciences, University of \\ Mazandaran, Babolsar, IR Iran. Tel/Fax: +98-1125242161, E-mail: acolagar@yahoo.com, ahcolagar@umz.ac.ir.
}

Received: May 18, 2012; Revised: January 07, 2013; Accepted: April 30, 2013

Background: Sperm dysfunction caused by reactive oxygen species (ROSs) is one of the major causes of infertility in men, which leads to, lipid peroxidation (LPO) and the formation of stable peroxidation products like Malondialdehyde (MDA) in seminal plasma. MDA is effective factor in reducing fertility.

Objectives: The aim of this study is to determine two biochemical markers of oxidative stress; TAC and MDA, and them correlation to quality-quantity factors in Asthenoteratospermic and Oligoashenoteratospermic men.

Patients and Methods: A total of 42 semen samples including: 15 samples normospermic as control group, 12 Asthenoteratospermic and 15 oligoasthenoteratospermic were collected from Babol IVF center; Iran. Semen analysis was performed according to WHO (1999) guidelines. Seminal plasma TAC and MDA levels in all patients were measured by TBARs and FRAP methods, respectively.

Results: Seminal plasma TAC level in normospermic men was significantly higher than asthenoteratospermic men $(\mathrm{P}<0.001)$ and oligasthenoteratospermic men $(\mathrm{P}<0.001)$ and had posetive correlation with sperm count, motility and morphology. In contrast MDA levels in normospermic men were significantly lower than in asthenoteratospermic men $(\mathrm{P}=0.049)$ and oligoasthenoteratospermic men $(\mathrm{P}=0.001)$ and had negative correlation with sperm count, motility and morphology.

Conclusions: These results suggest that lipid peroxidation and decreasing total antioxidant capacity lead to low motility; morphology and sperm count in spermatozoa of astheno-and oligoastheno-teratospermic men. Therefore, evaluation of oxidative status and antioxidant defenses system may be as a useful tool for diagnosis and treatment of male infertility especially in idiopathic male infertility.

Keywords: Lipid Peroxidation; Malondialdehyde; Reactive Oxygen Species

\section{Introduction}

Oxidative stress is an important factor which influences fertility potential of spermatozoa by lipid peroxidation which may result in sperm dysfunction. Sperm count and sperm motility are fundamental parameters that ascertain the functional ability of spermatozoa (1). Decreased the sperm motility (asthenozoospermia) is considered to associate with the infertility of a significant number of males, and many cases of reduction in the sperm motility are not completely understood. Many factors can affect sperm motility, but they are still not clear. One of them that potentially causes asthenozoospermia is oxidative stress induced by ROS (2-4). The most common ROS that have potential significance in reproductive biology, include the superoxide anion $\left(\mathrm{O}^{-}\right)$, hydrogen peroxide $(\mathrm{H} 2 \mathrm{O} 2)$, the peroxyl $\left(\mathrm{ROO}^{-}\right)$and the hydroxyl $\left(\mathrm{OH}^{-}\right)$radicals $(5,6)$. Reactive oxygen species (ROS) has both physiological and pathological roles in male infertility. The physiological level of ROS plays a crucial role in processes such as maturation, capacitation, acrosomal reactions, and fertilization (7-10). On the other hand, pathological levels of ROS, which can originate from endogenous sources such as leukocytes $(11,12)$ and immature/abnormal spermatozoa $(9,12)$ or from exogenous sources such as environmental factors (e.g. cigarette smoking, alcohol) (13, 14) can be potentially toxic for spermatozoan function due to the peroxidation of high polyunsaturated fatty acids (PUFA) within the plasma membrane of spermatozoa $(5,11,15)$ Increased ROS levels also have been associated with reduction in the sperm motility (16-18). However, the link between ROS and reduced motility in spermatozoa is not fully understood. Thus, many hypotheses have been proposed to explain it.

One hypothesis is that $\mathrm{H} 2 \mathrm{O} 2$ can diffuse across the cell membrane into the cytoplasm and inhibit the activity of enzymes such as glucose-6-phosphate dehydrogenase (G6PD). This enzyme controls the rate of glucose flux via the hexose monophosphate shunt which in turn, controls the intracellular availability of nicotinamide

Implication for health policy/practice/research/medical education:

Lipid peroxidation and decreasing total antioxidant capacity lead to low motility, morphology and sperm count in spermatozoa of astheno - and oligoastheno - teratospermic men.

Copyright (C) 2013, Iranian Red Crescent Medical Journal; Licensee KowsarKowsar Ltd. This is an Open Access article distributed under the terms of the Creative Commons Attribution License (http://creativecommons.org/licenses/by/3.0), which permits unrestricted use, distribution, and reproduction in any medium, provided the original work is properly cited. 
adenine dinucleotide phosphate (NADPH). This in turn is used as a source of electrons by spermatozoa to fuel the generation of ROS by an enzyme system known as NADPH oxidase (19). Inhibition of G6PD leads to a decrease in the availability of NADPH and a concomitant accumulation of oxidized glutathione and reduced glutathione. This can reduce the antioxidant defenses system of the spermatozoa and increase membrane phospholipids peroxidation (20). Another hypothesis involves a series of cascade chemical reactions that result in a decrease in axonemal protein phosphorylation and reduce sperm motility, both of which are associated with a reduction in membrane fluidity and sperm-oocyte fusion (21). Malondialdehyde (MDA) is one of the reactive and mutagenic aldehyde products of lipid peroxidation in seminal plasma (22) Toxic lipid peroxides are known to cause different impairments of sperm cells and may play a main role in the etiology of male infertility. Malondialdehyde (MDA) is an indicator of lipid peroxidation which may be a diagnostic tool for the analysis of infertility $(23,24)$.

\section{Objectives}

We determined the correlation between the TAC and MDA concentration with the motility, morphology and sperm count of spermatozoa in asthenoteratospermic and oligoasthenoteratospermic in comparison with control men.

\section{Patients and Methods}

Forty-five semen samples from men in the age range 24 - 38 years attending the infertility clinic at Fatemeh Zahra Hospital (Babol, Iran) were collected into sterile containers after a period for 2-3 days of abstinence. Semen specimens were allowed to liquefy at $37^{\circ} \mathrm{C}$ for $30 \mathrm{~min}$. Routine analysis of semen was performed within 1 hour according to World Health Organization guidelines (25). A hematoxylin-eosin (H\&E) staining method was used for determination of the percent normal morphology of spermatozoa. Samples were then classified as "normozoospermic", "asthenotratospermic" (with motility <50\% and morphology $<14 \%$ ) and "oligoasthenoteratospermic "(with Sperm concentration < 20 million per ml, motility $<50 \%$ and morphology $<14 \%$ ). Morphology of the spermatozoa was assessed using Kruger's criteria that morphology $<14 \%$ is considered abnormal (26).

\subsection{Measurement of TAC}

Semen samples were centrifuged at $14000 \times \mathrm{g}$ for $7 \mathrm{~min}$. after centrifugation, supernatants were diluted 1:10 v/v in distilled water. TAC was then evaluated using ferricreducing ability of plasma (FRAP) according to method of Benzie (1996) (27) with slightly modifications. This method shows the ability of seminal plasma antioxidants to reduce ferric-tripyridyltriazine $\left(\mathrm{Fe}^{3+}-\mathrm{TPTZ}\right)$ to a ferrous form $\left(\mathrm{Fe}^{2+}\right)$. For TAC measurement, the working FRAP reagent was prepared by mixing $10 \mathrm{vol}$. of 300 $\mathrm{mmol} / \mathrm{l}$ acetate buffer; $\mathrm{pH} 3.6$ with 1 vol. of $10 \mathrm{mmol} / \mathrm{l} 2$, 4, 6,-tripyridyl-s-triazine in $40 \mathrm{mmol} / \mathrm{l} \mathrm{HCl}$ with one volume of $20 \mathrm{mmol} / \mathrm{l} \mathrm{FeCl3.6H2O}$. Then, $1.5 \mathrm{ml}$ of the working FRAP reagent was aliquoted into a glass tube and warmed to $37^{\circ} \mathrm{C}$ for 5 minutes. Subsequently, $50 \mu \mathrm{L}$ of plasma and $50 \mu \mathrm{L}$ of distilled water (reagent-free) as well as $50 \mu \mathrm{L}$ of each of the standard solutions (FeSO4.7H2O; 1000, 500, $250,125 \mu \mathrm{M}$ ) were added to $1.5 \mathrm{~mL}$ FRAP reagent and heated to $37^{\circ} \mathrm{C}$ for 10 minutes. Absorbance was measured at $593 \mathrm{~nm}$ using a spectrophotometer (UV-visible). The final results were expressed as $\mu \mathrm{M} / \mathrm{l} .6$.

\subsection{Measurement of MDA}

Seminal MDA levels were determined according to the method as described by Rao et al. (28), which was slightly modified by Gholinezhad and Hosseinzadeh Colagar (6).

\subsection{Statistical Analysis}

Data are presented as mean \pm S.D. An independent t-test using SPSS 16 for Windows software (SPSS Incorporated Chicago, IL, USA) was considered to analyze data collected. A linear regression (Spearman) model was applied to the relationship of MDA levels and TAC with the motility and morphology of spermatozoa. In all cases, $\mathrm{P}<0.05$ was considered statistically significant.

Table 1. Standard Semen Parameters Analysis in Healthy, Asthenoteratospermic and Oligoasthenoteratospermic Men

\begin{tabular}{|c|c|c|c|}
\hline Semen Parameters ${ }^{a}$ & $\mathrm{C}^{\mathrm{b}}$ & $\mathbf{A T}^{\mathrm{b}}$ & $\mathbf{O A T}^{\mathrm{b}}$ \\
\hline Volume (mL) & $3.067 \pm 0.88$ & $2.29 \pm 1.01^{\mathrm{e}}$ & $2.60 \pm 0.92$ \\
\hline Sperm count $(\times 106 / \mathrm{mL})$ & $96.67 \pm 8.16$ & $47.75 \pm 22.97^{C}$ & $5.07 \pm 2.4^{\mathrm{C}}$ \\
\hline Total sperm $(\times 106)$ & $292.33 \pm 78.03$ & $108.0 \pm 66.68^{C}$ & $12.71 \pm 6.98^{C}$ \\
\hline Sperm motility, No. (\%) & $62.66 \pm 7.98$ & $33.50 \pm 4.66^{\mathrm{C}}$ & $20.53 \pm 14.54^{C}$ \\
\hline Sperm morphology, No. (\%) & $27.86 \pm 9.34$ & $7.58 \pm 2.61^{\mathrm{C}}$ & $6.5 \pm 3.22^{C}$ \\
\hline
\end{tabular}




\section{Results}

Semen analysis was done according to WHO guidelines 25.The concentration of MDA and the TAC in seminal plasma was measured by TBARs and FRAP methods, respectively.

\subsection{Semen Analysis}

The parameters investigated in semen samples are shown in Table 1.

All parameters (count, total count, motility and morphology of spermatozoa) in control men were strongly significant $(\mathrm{P}<0.001)$ in comparison with patient groups (Figure 1).

Figure 1. Comparison of Percentage of Motility, Morphology and Sperm Count $(\times 106 / \mathrm{Ml})$ Between Control (C) and Patient Groups (AT, OAT)

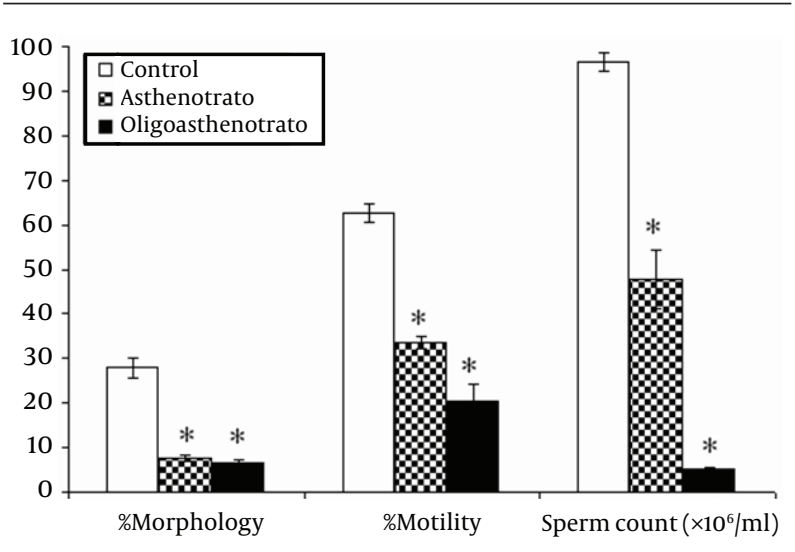

Percentage of normal sperm morphology between patient groups was significant $(\mathrm{P}=0.006)$. Error bar, representing SEM. $\mathrm{P}<0.05$ was considered statistically significant, ${ }^{*} \mathrm{P}<0.001$

Semen volume in asthenotratospermic was also significantly lower than in healthy men $(\mathrm{P}<0.05$ respectively) and in oligoasthenoteratospermic was lower than in healthy men $(\mathrm{P}=0.13)$ but this relation was not significant.

\subsection{TAC and MDA Assays}

Comparison of the TAC and MDA levels are shown in Table 2.

MDA levels in asthenoteratospermic and oligoasthe- noteratospermic were significantly higher than in healthy men $(\mathrm{P}=0.049$ and $\mathrm{P}=0.001$, respectively), but a significant difference between the patient groups was not observed. In contrast, the TAC in asthenoteratospermic and oligoasthenoteratospermic was significantly lower than in healthy men $(\mathrm{P}<0.001$, respectively), but a significant difference between the TAC in the patient groups was not seen. We also observed a negative correlation between MDA levels with the motility $(\mathrm{P}=0.048)$, morphology $(\mathrm{P}=0.001)$ and sperm count $(\mathrm{P}=0.001)$ of spermatozoa, but a positive correlation between the TAC with motility $(\mathrm{P}<0.001)$, morphology $(\mathrm{P}<0.001)$ and sperm count $(\mathrm{P}<$ $0.001)$ in asthenoteratospermic and oligoasthenoteratospermic men. There was a negative correlation between MDA levels with the sperm count $(\times 106 / \mathrm{ml})(\mathrm{P}=0.001)$ and a positive correlation between the TAC with the sperm count $(\times 106 / \mathrm{ml})(\mathrm{P}<0.001)$ (Figure 2,3 and 4$)$.

\section{Discussion}

Oxidative stress (OS) as a result of an inappropriate balance between oxidants and antioxidants in the semen can lead to sperm damage, impairs the structure and function of spermatozoa and eventually male infertility $(29,30)$ OS results from Reactive Oxygen Species (ROS) or free radicals. In physiological conditions, ROS are required for crucial aspects of sperm function, in pathological conditions, excessive levels of ROS can negatively affect sperm quality (31). Sperm plasma membrane is particularly susceptible to lipid peroxidation by ROS due to the existence of high concentration of polyunsaturated fatty acids. Lipid peroxidation can lead to loss of membrane fluidity and integrity, as a result of this, reduces sperm-oocyte fusion. Furthermore, they can attack DNA by inducing base modifications, DNA strand breaks, DNA cross-links, and chromosomal rearrangements (32). Overproduction of ROS is associated with defective sperm function $(33,34)$. It is necessary a fine balance between ROS production and recycling, for successful spermatogenesis. There are some potential origins of ROS such as, seminal leukocytes with positive proxidase, immature, morphologically abnormal sperm, can be a cause of male infertility (35). Exposing the spermatozoa to ROS, causes DNA damage and lipid peroxidation (36). High lipid peroxidation may lead to reduce acrosomal reaction, fertilization (37), and sperm oocyte fusion.

Table 2. Comparison of TAC and MDA Concentration in Healthy, Asthenoteratospermic and Oligoasthenoteratospermic Men ${ }^{\mathrm{a}}$

\begin{tabular}{lllllcc}
\hline & \multicolumn{5}{c}{ P value } \\
\hline Variables & C & AT & OAT & C vs. AT & C vs. OAT & AT vs. OAT \\
TAC $(\mu \mathbf{M} / \mathbf{l})$ & $3239 \pm 562.25$ & $1916.4 \pm 575.39$ & $1896.7 \pm 650.86$ & $<0.001$ & $<0.001$ & 0.103 \\
MDA $(\mathbf{n m o l} / \mathbf{m l})$ & $0.569 \pm 0.20$ & $0.889 \pm 0.25$ & $0.932 \pm 0.31$ & 0.049 & 0.001 & 0.473 \\
\hline
\end{tabular}

\footnotetext{
${ }^{\mathrm{a}}$ Values expressed as mean \pm SD and $\mathrm{P}<0.05$ was considered statistically significant.
} 
Figure 2. Correlation of Sperm Morphology With Total Antioxidant Capacity/TAC
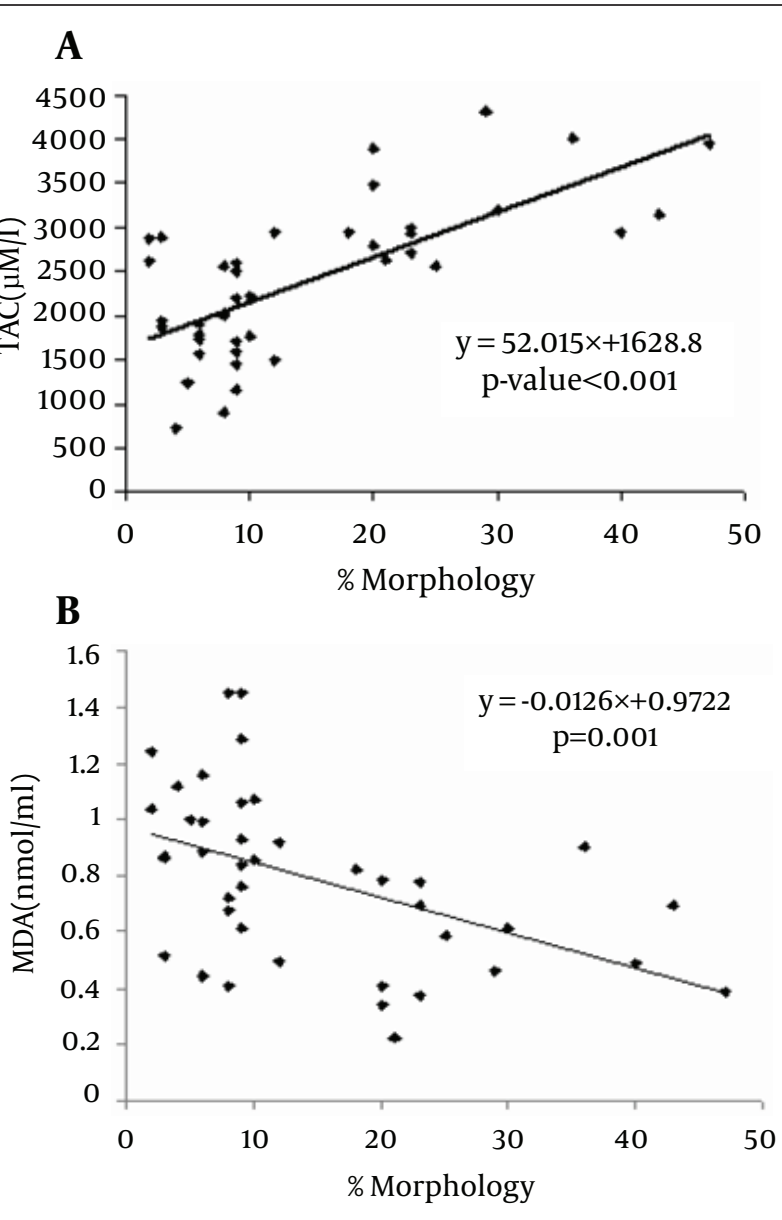

A) Malondialdehyde/MDA, B).Correlation is Significant at the $\mathrm{P}<0.05$ Level (2-Tailed).

Malondialdehyde can be used as a marker of oxidative stress and a potential marker for predicting assisted reproductive techniques (ART) outcomes $(38,39)$. Several studies have shown that lipid peroxidation impacts the sperm concentration, motility, morphology and associated with poor sperm quality (40-42) Patel et al. observed (43) the negative correlation between the MDA levels and the normal sperm motility and morphology, so they suggested damaging effect of free radicals on sperm membrane integrity. Asbagh et al. (44) showed that there was significant association between semen MDA and abnormal sperm morphology, and decrease semen TAC and weak sperm motility. Gholinezhad and Hosseinzadeh Colagar6 showed a negative correlation between MDA levels and the motility and morphology of spermatozoa and a positive correlation between the TAC reflected by motility and morphology of spermatozoa in asthenoteratospermic smokers and non-smokers in comparison with a control group. In this study,
Figure 3. The Correlations of the Sperm Motility with TAC

\section{A}

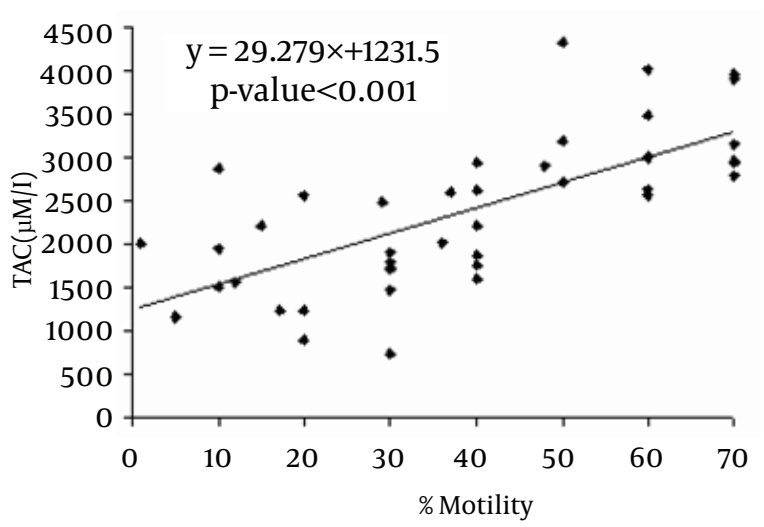

B

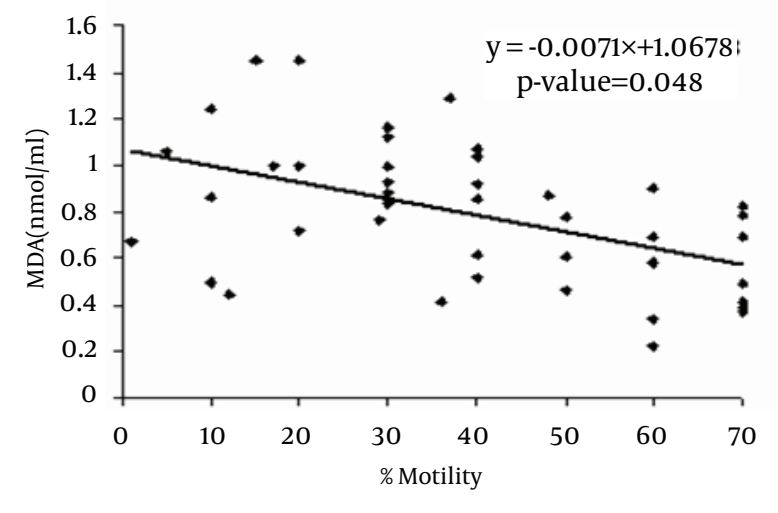

A) MDA, B) Correlation is significant at $\mathrm{P}<0.05$ level (2-tailed).

we found Seminal TAC in asthenoteratospermic and oligoasthenoteratospermic men was significantly lower than in healthy ( $\mathrm{P}<0.001$ respectively). Our results are in accordance with the study of Badade et al. (45), Koca et al. (46) and Khosrowbeygi et al. (47) Badade et al. (45) reported that TAC levels significantly lower in infertile men compared to fertile men. Khosrowbeeygi et al. (47) showed that TAC levels significantly lower in the asthenospermic, asthenoteratospermic and oligoasthenoteratospermic versus control group. Moreover, MDA levels in the control group were significantly lower than in asthenoteratospermic and oligoasthenoteratospermic patients $(\mathrm{P}=0.049$ and $\mathrm{P}$ $=0.001$, respectively). A negative correlation between MDA levels with Sperm count, motility and normal morphology also a positive correlation in the TAC with Sperm count, motility and normal morphology between control group and asthenoteratospermic and oligoasthenoteratospermic men was observed. Our results of MDA are concurrent with Kobayashi et al. (48), 
Figure 4. Correlations of Sperm Count $(\times 106 / \mathrm{ml})$ With TAC

A

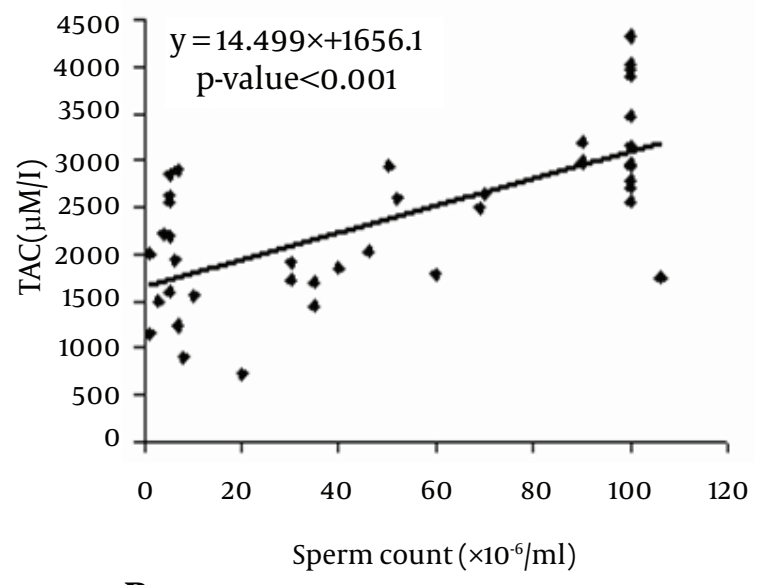

B

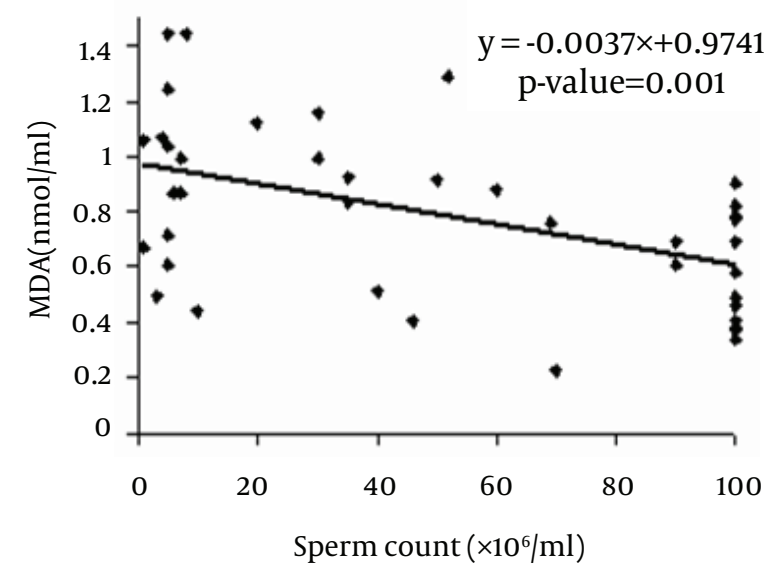

A) MDA, B) Correlation is significant at $\mathrm{P}<0.05$ level (2-tailed).

Parineeta et al. (49) and Badade et al. (45) Kobayashi et al. (47) demonstrated high seminal MDA level in patients with oligoasthenoteratozoospermia. More over our results are in contrast with Suleiman et al. (50) They showed that MDA level in the seminal plasma was not correlated with the sperm concentration and motility.

Nabil et al. (51) observed MDA concentration in oligozoospermic and azoospermic men was significantly higher than normozoospermic while glutathione, ascorbic acid and total antioxidant status were significantly reduced in oligozoospermic and azoospermic men compared to normozoospermic group. Our study suggests, high ROS levels in semen lead to lipid peroxidation represented by MDA in semen, which may contribute to low motility, morphology and sperm count in the spermatozoa of asthenoteratospermic and oligoasthenoteratospermic patients. It is also important to determine the antioxidant status of semen as it is a major defense mechanism. The increase in malondialdehyde, and decrease in TAC levels in astheno- and oligoastheno-teratospermic men may have significant role in the etiology of sperm dysfunction. Positive correlation of TAC with sperm count, sperm motility and morphology, and negative correlation of sperm parameters with MDA indicate oxidative stress has a harmful effect in male infertility. Thus evaluation of seminal MDA and TAC could be beneficial diagnostic tool for defining sperm fertilization potential. These parameters could help in distinction and treatment of male infertility especially in idiopathic cases.

\section{Acknowledgements}

This is part of MSc. dissertation. We wish to thank Zahra Infertility and Health Reproductive Research Center (Babol University of Medical Sciences, Babol) for sampling, doing the laboratory tests and other supports.

\section{Authors' Contribution}

No distribution of duties have been declared.

\section{Financial Disclosure}

The authors declare that there is no conflict of interest.

\section{Funding Support}

None Declared

\section{References}

1. Badade ZG, More K, Narshetty J. Oxidative stress adversely affects spermatogenesis in male infertility. Biomed Res. 2011a;22(3):323-8.

2. Conquer JA, Martin JB, Tummon I, Watson L, Tekpetey F. Fatty acid analysis of blood serum, seminal plasma, and spermatozoa of normozoospermic vs. Asthernozoospermic males. Lipids. 1999;34(8):793-9.

3. Gagnon C, Lamirande E, Hamamah S, Olivennes F, Mieusset R, Frydman R. Extrinsic Factors Affecting Sperm Motility: Immunological and Infectious Factors and Reactive Oxygen Species. In: Gagnon C, Lamirande E, Hamamah S, Olivennes F, Mieusset R, Frydman R, editors.Male Sterility and Motility Disorders.Springer New York; 1999. p. 37-44.

4. Zarghami Nosratollah, Khosrowbeygi Ali. Seminal plasma levels of 15-F2 $\alpha$-isoprostane, malondialdehyde and total homocysteine in normozoospermic and asthenozoospermic males. Indian $J$ Clin Biochem. 2005;20(2):86-91.

5. Maneesh M, Jayalekshmi H. Role of reactive oxygen species and antioxidants on pathophysiology of male reproduction. Indian J Clin Biochem. 2006;21(2):80-89.

6. Gholinezhad Chari M, Hosseinzadeh Colagar A. Seminal plasma lipid peroxidation, total antioxidant capacity, and cigarette smoking in asthenoteratospermic men. J Men's Health. 2011;8(1):43-9.

7. Agarwal A, Saleh RA. Role of oxidants in male infertility: rationale, significance, and treatment. Urol Clin North Am. 2002;29(4):81727.

8. Agarwal A, Saleh RA, Bedaiwy MA. Role of reactive oxygen species in the pathophysiology of human reproduction. Fertil Steril. 2003;79(4):829-43.

9. Gil-Guzman E, Ollero M, Lopez MC, Sharma RK, Alvarez JG, Thomas AJ, et al. Differential production of reactive oxygen species by subsets of human spermatozoa at different stages of maturation. Hum Reprod. 2001;16(9):1922-30.

10. Pasqualotto FF, Sharma RK, Nelson DR, Thomas AJ, Agarwal A. Relationship between oxidative stress, semen characteristics, and 
clinical diagnosis in men undergoing infertility investigation. Fertil Steril. 2000;73(3):459-64.

11. Sikka SC, Rajasekaran M, Hellstrom WJ. Role of oxidative stress and antioxidants in male infertility.J Androl. 1995;16(6):464-8.

12. Saleh RA, Agarwal A. Oxidative stress and male infertility: from research bench to clinical practice. J Androl. 2002;23(6):737-52.

13. Cocuzza Marcello, Sikka SureshC, Athayde KellyS, Agarwal Ashok Clinical relevance of oxidative stress and sperm chromatin damage in male infertility: an evidence based analysis. Int braz $j$ urol. 2007;33:603-21.

14. Saleh RA, Agarwal A, Sharma RK, Nelson DR, Thomas AJ. Effect of cigarette smoking on levels of seminal oxidative stress in infertile men: a prospective study. Fertil Steril. 2002;78(3):491-9.

15. Aitken RJ, Clarkson JS, Fishel S. Generation of reactive oxygen species, lipid peroxidation, and human sperm function. Biol of Reprod.1989;41(1):183-97.

16. Lenzi A, Lombardo F, Gandini L, Alfano P, Dondero F. Computer assisted sperm motility analysis at the moment of induced pregnancy during gonadotropin treatment for hypogonadotropic hypogonadism. J Endocrinol Invest. 1993;16(9):683-6.

17. Agarwal A, Ikemoto I, Loughlin KR. Relationship of sperm parameters with levels of reactive oxygen species in semen specimens. J Urol.1994;152(1):107-10.

18. Armstrong JS, Rajasekaran M, Chamulitrat W, Gatti P, Hellstrom WJ, Sikka SC. Characterization of reactive oxygen species induced effects on human spermatozoa movement and energy metabolism. Free Radic Biol Med.1999;26(7-8):869-80.

19. Aitken RJ, Fisher HM, Fulton N, Gomez E, Knox W, Lewis B, et al. Reactive oxygen species generation by human spermatozoa is induced by exogenous NADPH and inhibited by the flavoprotein inhibitors diphenylene iodonium and quinacrine. Mol Reprod Dev. 1997;47(4):468-82.

20. Griveau JF, Dumont E, Renard P, Callegari JP, Le Lannou D. Reactive oxygen species, lipid peroxidation and enzymatic defence systems in human spermatozoa. J Reprod Fertil. 1995;103(1):17-26.

21. de Lamirande E, Gagnon C. Reactive oxygen species and human spermatozoa. II. Depletion of adenosine triphosphate plays an important role in the inhibition of sperm motility. J Androl. 1992;13(5):379-86.

22. ShangXJ, Li K, YeZQ, Chen YG, Yu X, Huang YF. Analysis of lipid peroxidative levels in seminal plasma of infertile men by high-performance liquid chromatography. Arch Androl. 2004;50(6):411-6.

23. Tavilani H, Doosti M, Saeidi H. Malondialdehyde levels in sperm and seminal plasma of asthenozoospermic and its relationship with semen parameters. Clin Chim Acta. 2005;356(1-2):199-203.

24. Tavilani H, Goodarzi MT, Vaisi-Raygani A, Salimi S, Hassanzadeh T. Activity of antioxidant enzymes in seminal plasma and their relationship with lipid peroxidation of spermatozoa. Int braz $j$ urol. 2008;34:485-91.

25. World Health Organization. WHO Laboratory Manual for the Examination of Human Semen and Sperm-cervical Mucus Interaction. 1999.

26. Makker K, Agarwal A, Sharma R. Oxidative stress \& male infertility. Indian J Med Res. 2009;129(4):357-67.

27. Benzie IFF. Lipid peroxidation: A review of causes, consequences, measurement and dietary influences. Int J Food Sc and Nutr. 1996;47(3):233-61

28. Rao B, Soufir JC, Martin M, David G. Lipid peroxidation in human spermatozoa as relatd to midpiece abnormalities and motility. Gamete Res. 1989;24(2):127-34.

29. Kruger TF, Menkveld R, Stander FS, Lombard CJ, Van der Merwe JP, van Zyl JA, et al. Sperm morphologic features as a prognostic factor in in vitro fertilization. Fertil Steril. 1986;46(6):1118-23.

30. Agarwal A, Varghese AC, Sharma RK. Markers of oxidative stress and sperm chromatin integrity. Methods Mol Biol. 2009;590:377402.

31. Kefer JC, Agarwal A, Sabanegh E. Role of antioxidants in the treatment of male infertility. Int JUrol 2009;16(5):449-57.
32. Duru NK, Morshedi M, Oehninger S. Effects of hydrogen peroxide on DNA and plasma membrane integrity of human spermatozoa. Fertil Steril. 2000;74(6):1200-7.

33. Iwasaki A, Gagnon C. Formation of reactive oxygen species in spermatozoa of infertile patients. Fertil Steril. 1992;57(2):409-16.

34. Mazzilli F, Rossi T, Marchesini M, Ronconi C, Dondero F. Superoxide anion in human semen related to seminal parameters and clinical aspects. Fertil Steril. 1994;62(4):862-8.

35. Plante M, de Lamirande E, Gagnon C. Reactive oxygen species released by activated neutrophils, but not by deficient spermatozoa, are sufficient to affect normal sperm motility. Fertil Steril. 1994;62(2):387-93.

36. Potts RJ, Notarianni LJ, Jefferies TM. Seminal plasma reduces exogenous oxidative damage to human sperm, determined by the measurement of DNA strand breaks and lipid peroxidation. $\mathrm{Mu}$ tat Res. 2000;447(2):249-56.

37. Aitken RJ, Warner P, Best FSM, Templeton AA, Djahanbakhch O, Mortimer D, et al. The predictability of subnormal penetrating capacity of sperm in cases of unexplained infertility. Int J Androl. 1983;6(3):212-20.

38. Oral Ozay, Kutlu Tayfun, Aksoy Evrim, Fıçıcıoğlu Cem, Uslu Hüsamettin, Tuğrul Semih. The Effects of Oxidative Stress on Outcomes of Assisted Reproductive Techniques. J Assist Reprod Genet. 2006;23(2):81-5.

39. Jedrzejczak P, Fraczek M, Szumala-Kakol A, Taszarek-Hauke G, Pawelczyk L, Kurpisz M. Consequences of semen inflammation and lipid peroxidation on fertilization capacity of spermatozoa in in vitro conditions. Int J Androl. 2005;28(5):275-83.

40. Hsieh YY, Chang C, Lin CS. Seminal malondialdehyde concentration but not glutathione peroxidase activity is negatively correlated with seminal concentration and motility. Int J Biol Sci. 2006;2(1):23.

41. Huang YL, Tseng WC, Cheng SY, Lin TH. Trace elements and lipid peroxidation in human seminal plasma. Biol Trace Elem Res. 2000;76(3):207-15.

42. Gomez E, Irvine DS, Aitken RJ. Evaluation of a spectrophotometric assay for the measurement of malondialdehyde and 4-hydroxyalkenals in human spermatozoa: relationships with semen quality and sperm function. Int J Androl. 1998;21(2):81-94.

43. Patel S, Panda S, Nanda R, Mangaraj M, Mohapatra PC. Influence of oxidants and anti-oxidants on semen parameters in infertile males. J Indian Med Assoc. 2009;107(2):78-80.

44. Akbari Asbagh F, Mostafavi E, Hamdi K, Azmodeh O, Ghasemynejad A, Moshtaghi J, et al. Relation of serum and semen malondialdehyde and total anti-oxidants with sperm parameters in infertile men. Am J Immunol. 2010;6(3):43-9.

45. Badade G, More K, Narshetty G, Badade Vandana Z. Human seminal oxidative stress: correlation with antioxidants and sperm quality parameters. Ann Biol Res. 2011b;2:351-9.

46. Koca YOOLCMUSBN, Özdal ÖL, Celik M, Ünal S, Balaban N. Antioxidant activity of seminal plasma in fertile and infertile men. Arch Androl.2003;49(5):355-9.

47. Khosrowbeygi Ali, Zarghami Nosratollah, Deldar Yaghoub. Correlation between sperm quality parameters and seminal plasma antioxidants status. Iran J Reprod Med. 2012;2(2):58-64.

48. Kobayashi T, Miyazaki T, Natori M, Nozawa S. Protective role of superoxide dismutase in human sperm motility: superoxide dismutase activity and lipid peroxide in human seminal plasma and spermatozoa. Hum Reprod. 1991;6(7):987-91.

49. Parineeta MS, Zunjarrao GB, Madhuri SK, Deepak A. Seminal Malondialdehyde Concentration And Superoxide Dismutase, Catalase Activity In Male Infertility. Internet J Health. 2009;11(2).

50. Suleiman SA, Ali ME, Zaki ZM, el-Malik EM, Nasr MA. Lipid peroxidation and human sperm motility: protective role of vitamin E.J Androl.1996;17(5):530-7.

51. Nabil H, Moemen LA, Elela MHA. Studying the levels of malondialdehyde and antioxidant parameters in normal and abnormal human seminal plasma. Aust J Basic Appl Sci. 2008;2(3):773-8. 